\title{
SIMULATION OF THE PROCESS OF FLOW SEPARATION AT THE ENTRANCE OF SQUARE ASPIRATING PORT
}

\author{
Olga A. Averkova, Ilya V. Kryukov, \\ Kirill V. Plotnikov, Elena I. Tolmacheva and Ilya V. Khodakov \\ Belgorod State Technological University Named After V.G. Shoukhov, 308012, Kostykova str., 46, Russia
}

Received 2014-06-27; Revised 2014-09-08; Accepted 2014-10-17

\begin{abstract}
We consider the flow at the inlet to the suction square hole with sharp edges, which is located in an infinite space. The purpose of this study is to construct a mathematical model of flow separation at the entrance to square suction canal with sharp edges, located in infinite space, by using square vortex frameworks. As a part of ideology of the method of discrete vortices in the non-stationary quasiaxisymmetric formulation, we constructed the mathematical model of separated flow at the inlet to the square aspirating pipe and its software-algorithmic implementation. We have determined the velocity field at the entrance to suction channel and a line of flow separation. Determine the velocity field in typical cross-sections of the suction channel, dimensions of the efficient absorption, compression ratio of the jet. Were received analytical formulas for calculating separation surfaces current. Profiling the entrance opening of the suction hole detected on the outlines separation surface will improve the acoustic and aerodynamic properties of the exhaust systems. The obtained results can be useful for designing of local exhaust ventilation of reduced energy consumption.
\end{abstract}

Keywords: Flows Near of Suction Holes, Separated Flows, The Method of Discrete Vortices

\section{INTRODUCTION}

For trapping pollutants apply local ventilation devices of different forms (Logachev et al., 2013). Round suction holes fairly well studied. Is interesting to study square suction holes considering flow separation. Until now, this has not been done.

Separated flows at the inlet to the suction channels were considered in two-dimensional formulation: Plane and axisymmetric (Schinzinger and Laura, 2012; Averkova et al., 2012). Using the methods of complex variable theory is only possible for flat separated flows in multiply connected domains. The method of singular integral equations (Ginevsky and Zhelannikov, 2009; Marshall and Shuiqing, 2014) and in particular the method of discrete vortices allow you to extend the class of solved problems. By using the discrete vortex method
(Averkova et al., 2010; 2013b; 2013d) solved problems of separated flows at the entrance to circular tube with sharp edges (Averkova et al., 2013a), near-suction bells (Logachev et al., 2010.), at the entrance to the slit and round suction ports with screens (Averkova et al., 2014). For constructing discrete model were used infinitely thin straight and circular vortexes, the use of which does not allow to solve spatial problems. The purpose of this study is to construct a mathematical model of flow separation at the entrance to square suction canal with sharp edges, located in infinite space, by using square vortex frameworks.

\section{MATERIALS AND METHODS}

The problem consists in the definition of velocity field in the spectrum of action of a square semi-infinite Kostykova str., 46, Russia 
branch pipe (Fig. 1a), at a predetermined rate of absorption in the cross section, which is considerably remote from the entrance to the pipe. Throughout the aspirating section of $\mathrm{ABCD}$ the rate of absorption assumed to be constant and equal $v_{0}$. Disruption of the flow occurs from the sharp edge of EFMN. Condition of impermeability is executed on the pipe walls.

A discrete model is constructed as follows (Fig. 1b). The walls of the tube are discretized on square vortex frameworks (attached vortices) and calculated points, where the boundary conditions satisfy of impenetrability for normal velocity component. These points are located on the tube wall in the middle of between the vortex frameworks. The aspirating section is also divided into square vortex frameworks in the center of each contains calculated point where the normal component of the velocity is equal to the rate of absorption.

The influence of the vortex segment $A B$ with a constant rate $\mathrm{W}$ of turbulent layer at a point $M(x, y, z)$ is determined by the following formula:

$$
\begin{aligned}
& v=\frac{W}{4 \pi} \times \frac{A B \times A M}{|A B|^{2} \times|A M|^{2}-(A B \times A M)^{2}} \times \\
& \times\left(-\frac{A B \times B M}{|B M|}+\frac{A B \times A M}{|A M|}\right)
\end{aligned}
$$

where, $v$ the rate at the point of $M(x, y, z), A\left(a_{1}, a_{2}, a_{3}\right)$ and $B\left(b_{1}, b_{2}, b_{3}\right)$-tops of segment:

$$
\begin{aligned}
& A B=\left\{b_{1}-a_{1}, b_{2}-a_{2}, b_{3}-a_{3}\right\}, \\
& A M=\left\{x-a_{1}, y-a_{2}, z-a_{3}\right\}, \\
& A B \times A M=\left(b_{1}-a_{1}\right)\left(x-a_{1}\right)+\left(b_{2}-a_{2}\right)\left(y-a_{2}\right)+ \\
& +\left(b_{3}-a_{3}\right)\left(z-a_{3}\right), \\
& A B \times A M=\left(b_{1}-a_{1}\right)\left(x-b_{1}\right)+\left(b_{2}-a_{2}\right)\left(y-b_{2}\right)+ \\
& +\left(b_{3}-a_{3}\right)\left(z-b_{3}\right), \\
& A B \times A M=\left|\begin{array}{ccc}
i & j & k \\
b_{1}-a_{1} & b_{2}-a_{2} & b_{3}-a_{3} \\
x-a_{1} & y-a_{2} & z-a_{3}
\end{array}\right| .
\end{aligned}
$$
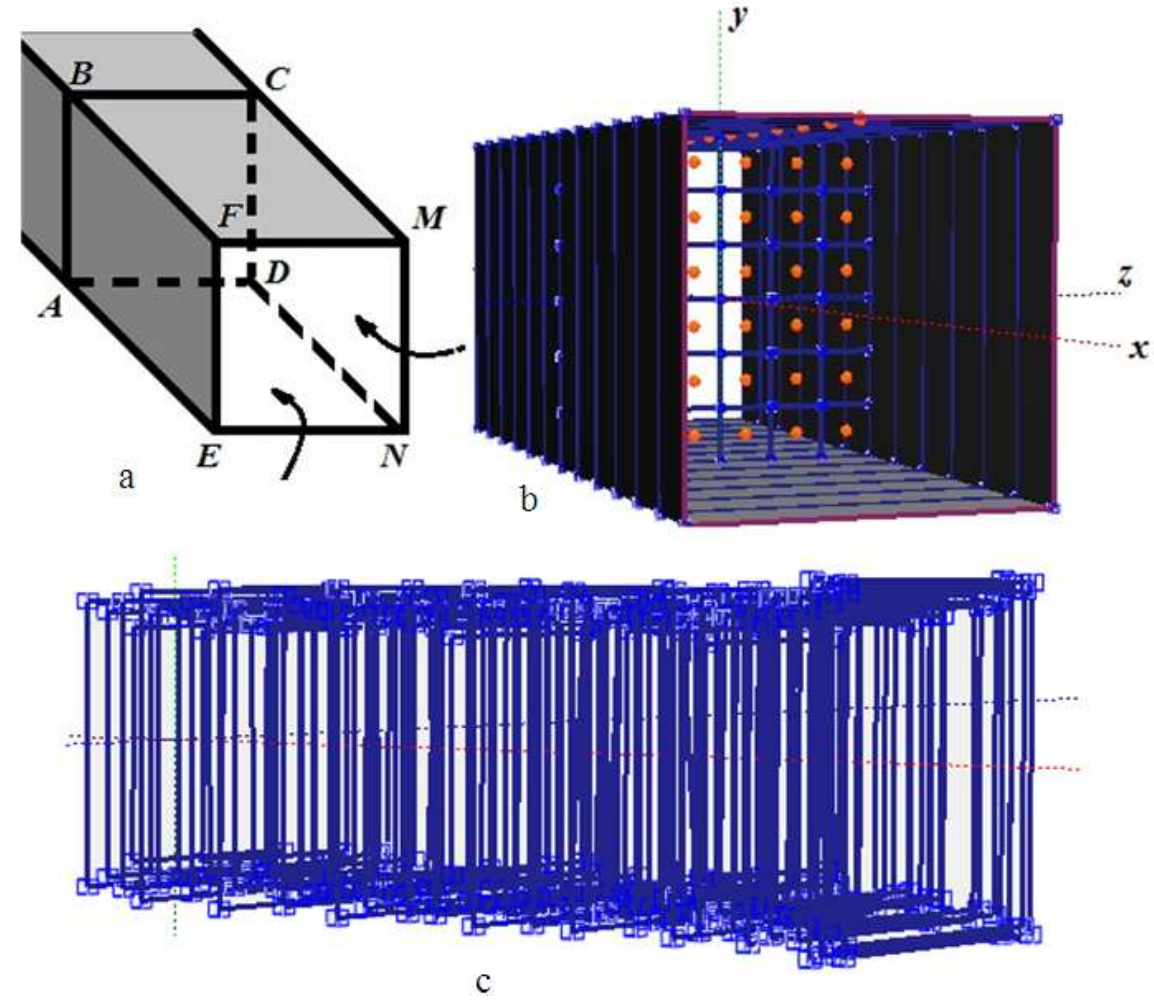

Fig. 1. The square branch pipe in an infinite space: (a) The flow scheme; (b) discrete model, (c) free square vortex frameworks, that are completely filled the area of flow inside the pipe 
For determining magnitude of velocity along a single direction $-n\left\{n_{1}, n_{2}, n_{3}\right\}$ of is necessary to find the velocity components $\left\{v_{1}, v_{2}, v_{3}\right\}$ using the formula (1) and then calculate the required speed:

$$
v_{n}=v_{1} n_{1}+v_{2} n_{2}+v_{3} n_{3}
$$

Let us introduce notations: $r_{1}, r_{2}, r_{3}, r_{4}$, - radius of the vectors of the vertices of the quadrilateral. The impact on any point of the vortex quadrangular frame with constant intensity $W$ is defined by the formula:

$$
\begin{aligned}
& v(x)=\frac{W}{4 \pi} \sum_{i=1}^{4}\left[\frac{\left[\left(r_{i+1}-r_{i}\right) \times r_{i}\right]}{\left|r_{i+1}-r_{i}\right|^{2}\left|r_{i}\right|^{2}-\left(\left(r_{i+1}-r_{i}\right) \times r_{i}\right)^{2}}\right. \\
& \left.\times\left(-\frac{\left(r_{i+1}-r_{i}\right) \times r_{i+1}}{\left|r_{i+1}\right|}+\frac{\left(r_{i+1}-r_{i}\right) \times r_{i}}{\left|r_{i}\right|}\right)\right]
\end{aligned}
$$

Here is assumed: $r_{5}=r_{1}$.

Let us introduce function of influence on any point $x$ $\left(x_{1}, x_{2}, x_{3}\right)$ of $k$-th frameworks of unit quadrangular vortex intensity as follows Equation 1:

$$
\begin{aligned}
& G(x, k)=\frac{1}{4 \pi} \sum_{i=1}^{4}\left[\frac{\left[\left(r_{i+1}^{k}-r_{i}^{k}\right) \times r_{i}^{k}\right]}{\left|r_{i+1}^{k}-r_{i}^{k}\right|^{2}\left|r_{i}^{k}\right|^{2}-\left(\left(r_{i+1}^{k}-r_{i}^{k}\right) \times r_{i}^{k}\right)^{2}}\right. \\
& \left.\times\left(-\frac{\left(r_{i+1}^{k}-r_{i}^{k}\right) \times r_{i+1}^{k}}{\left|r_{i+1}^{k}\right|}+\frac{\left(r_{i+1}^{k}-r_{i}^{k}\right) \times r_{i}^{k}}{\left|r_{i}^{k}\right|}\right)\right]
\end{aligned}
$$

where, $\quad r_{i}^{\mathrm{k}}=\left\{A_{1 i}^{\mathrm{k}}-x_{1}, A_{2 i}^{\mathrm{k}}-x_{2}, A_{3 i}^{\mathrm{k}}-x_{3}\right\}, \quad A_{i}^{k}\left(A_{1 i}^{k}, A_{2 i}^{k}, A_{3 i}^{k}\right) \quad i$-is vertex of $\mathrm{k}$-th of a quadrilateral framework.

Induced by framework $W(k)$ intensity of rate $v$ at $x$ along the direction is calculated using the scalar multiplication:

$$
v_{n}(x)=(G(x, k) \times n) W(k)
$$

Further we denote, $G^{p k}=\left(G\left(x^{p}, k\right) \times n\right)$, where $x^{p}$-p-th test point. Test points are located in the middle between of square vortex frameworks on the surface of pipe or in the center of vortex frameworks, located in the active section of pipe (Fig. 1b). Note that $\mathrm{W}$ intensity remains unchanged at all points of a square vortex framework.

Let us consider the initial time. At this moment of time there are only attached vortex frameworks (lying on the boundary of area). Intensity of these vortices $x^{p}$ is unknown. Normal component of the velocity at the point is calculated by adding together of all vortex frameworks Equation 2:

$v_{n}^{p}=\sum_{k=1}^{N} G^{p k} W^{k}$

where, $v_{n}^{p}=0$-speed in the $\mathrm{p}$-th test point (which is located in the center of the $\mathrm{p}$-th frame) on the surface of the pipe or $v_{n}^{p}=v_{0}$ in the active section. By changing the in an expression (2) number from 1 to $N$, we obtain a system of linear algebraic equations to determine the unknown intensities of vortex frameworks $W^{1}, W^{2}, \ldots W^{N}$. After solving the system of equations, the velocity at any point inside the area defined by the same formula (2), wherein instead of the $p$-th coordinate point of the calculated coordinates of this point are substituted.

At the time of $t=1 . \Delta t$ there occurs a disruption of the first free vortex framework from sharp edge of tube. Its position is determined from the formula Equation 3:

$$
x^{\prime}=x+v_{x} \times \Delta t, y^{\prime}=y+v_{y} \times \Delta t, z^{\prime}=z+v_{z} \times \Delta t
$$

where, $(x, y, z)$ the coordinates of its previous position, $\left(v_{x}, v_{y}, v_{z}\right)$-components of velocity vector find at this point using the formulas (1) and (2) at $n=\{1,0,0\}, n=\{0,1,0\}, n=\{0,0,1\}$ respectively. The intensity of this free vortex framework is equal to intensity of framework lying on a sharp edge of the pipe and over time does not change.

At this moment it is necessary to re-determine the intensity of attached vortex framework based on descended from sharp edge of a free vortex frame. The system of Equation 2 at this moment of time is converted to the following form Equation 4:

$$
\sum_{k=1}^{N} G^{p k} W^{k}=v_{n}^{p}-G^{p 1} \gamma^{1}, \quad p=1,2, \ldots N
$$

$G^{p 1}$-Influence function on the $p$-th test point of first free vortex framework of intensity $\gamma$. The next moment of time $t=2$. $\Delta t$ occurs descent of the second free vortex framework, the first-is shifting along direction of the flow. Positions of its vertices are defined by the formulas (3) and expression Equation 5:

$$
v_{n}(x)=\sum_{k=1}^{N} G^{k} W^{k}+G^{1} \gamma^{1}
$$


Unidentified circulation of attached vortex frameworks are determined by solving the transformed (4) of the following system equations:

$$
\sum_{k=1}^{N} G^{p k} W^{k}=v_{n}^{p}-\sum_{\tau=1}^{2} G^{p \tau} \gamma^{\tau}, \quad p=1,2, \ldots N
$$

Accordingly, the expression (5) is also converted into the form:

$$
v_{n}(x)=\sum_{k=1}^{N} G^{k} W^{k}+\sum_{\tau=1}^{2} G^{\tau} \gamma^{\tau}
$$

Thus, a new free vortex framework will go down into a flow at each moment of time. Location in space of vertices of "old" free frameworks will be determined by the formulas (3). In the time of $t=m$. $\Delta t$ the system for determining the unknown intensities of attached vortex frameworks will have a form Equation 6:

$$
\sum_{k=1}^{N} G^{p k} W^{k}=v^{p}-\sum_{\tau=1}^{m} G^{p \tau} \gamma^{\tau}, \quad p=1,2, \ldots N
$$

And the rate of speed at a given time in an interior point $x$ along a predetermined direction $n$ is determined by adding to this point all attached and free frameworks Equation 7:

$$
v_{n}(x)=\sum_{k=1}^{N} G^{k} W^{k}+\sum_{\tau=1}^{m} G^{\tau} \gamma^{\tau}
$$

where, $G^{k}$-influence function on point $x$ of $k$-th vortex framework; $W^{k}$ - its circulation, $G^{\tau}$-influence function on point $x$ of vortex framework, descended from a sharp edge at time of $t$. At a next moment of time occurs converging of new vortices, old ones are shifting in direction of flow, determined the unknown circulation of flow and until it reaches the predetermined target. At achievement a vortex framework of the active section of tube, it is removed from our consideration (Fig. 1c). Influence of vortices framework falling in sphere with center at a given point and with radius of discreteness $h$, is not considered. Radius of discreteness h typically is in the range of $0.88-0.99$ from the distance between adjacent vortex characteristics.

\section{RESULTS}

The calculations were carried out at the entrance to the square tube size of $0.2 \times 0.2 \mathrm{~m}$; length of pipe is $1 \mathrm{~m}$; active section of pipe is located in the middle that discretized by a set of 100 square frameworks; surface of tube was consisted of 100 square attached vortex frameworks (without framework of covering active section); radius of discreteness is $h=0.00495 \mathrm{~m}$ : time step is $\Delta_{t}=0.01 \mathrm{~s}$.

A velocity profile at the inlet of tube indicates that speed is minimal on the tube axis and then speed slightly increases to the walls (Fig. 2).

Tangential discontinuity of the velocity occurs at a distance of $0.6 \div 0.7$ a, that is a sharp change in the magnitude and direction of the velocity. Near the walls is observed vortex flow. The flow lines pulsate in process of time (Fig. 3). Value of width of effective suction in axial section passing through the centers of the pipe on opposite sides of the tube inlet, averaged in time, is equal B 0.82a. Upon entering the pipe, stream is compressed to $0.66 \mathrm{a}$. This occurs at a distance from the entrance to $0.8 \mathrm{a}$ pipe. For the flow lines in the coordinate system illustrated in Fig. 4 has a calculation formula Equation 8:

$$
x=1.72(|y|-1)^{2}-2.88(|y|-1)+0.82+0.76 \ln (|y|-0.66)
$$

Here are shown streamlines in a flatness passing through the axis of suction and through the middle of opposite sides. OX axis is directed along axis of the suction, the axis OY, OZ-through the middles of the opposite sides. All dimensions are related to half-side a square. It is clear that If a formula (8) change y to $\mathrm{z}$ we obtain flow lines in the plane of XOZ.

Detachable part of flow in the right side of coordinate system XYZ (Fig. 4) can be determined from the following Equation 9:

$$
\begin{aligned}
& (z \leq y \cap z \geq-y) \cup(z \geq y \cap z \leq-y) \Rightarrow \\
& x=1.72(|z|-1)^{2}-2.88(|z|-1)+0.82+0.76 \ln (|z|-0.66) \\
& (z \geq y \cap z \geq-y) \cup(z \leq y \cap z \leq-y) \Rightarrow \\
& x=1.72(|y|-1)^{2}-2.88(|y|-1)+0.82+0.76 \ln (|y|-0.66)
\end{aligned}
$$

\section{DISCUSSION}

Comparison of the values of axial velocity of air (Fig. 5) shows that the square intake port has less range than a round one. Along the ordinate axis was postponed magnitude of the velocity, divided by the rate of absorption $v_{0}$. Along the abscissa axis was postponed value of the distance from the entrance to the tube divided by the radius of the tube $\alpha$. 
Olga A. Averkova et al. / American Journal of Environmental Sciences 10 (5): 509-515, 2014

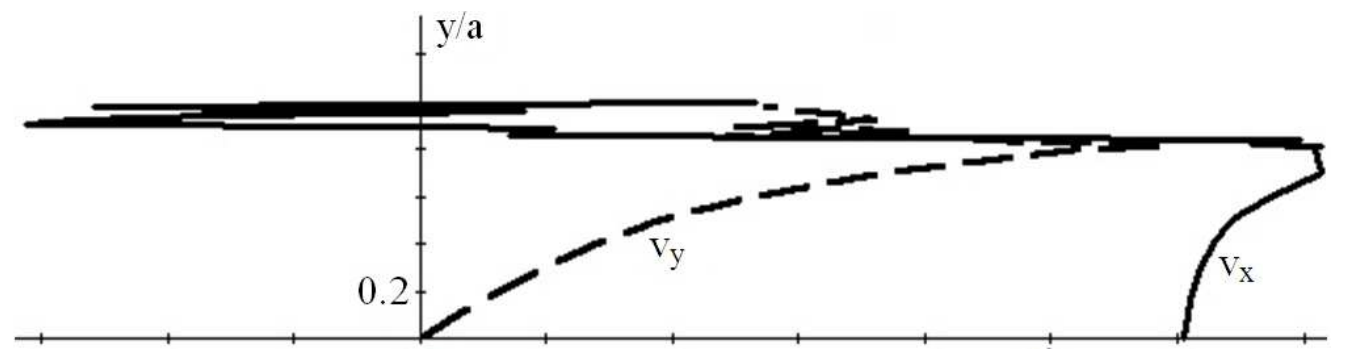

Fig. 2. The velocity profile at the entrance to square suction channel in the flatness passing through the middle of the opposite sides

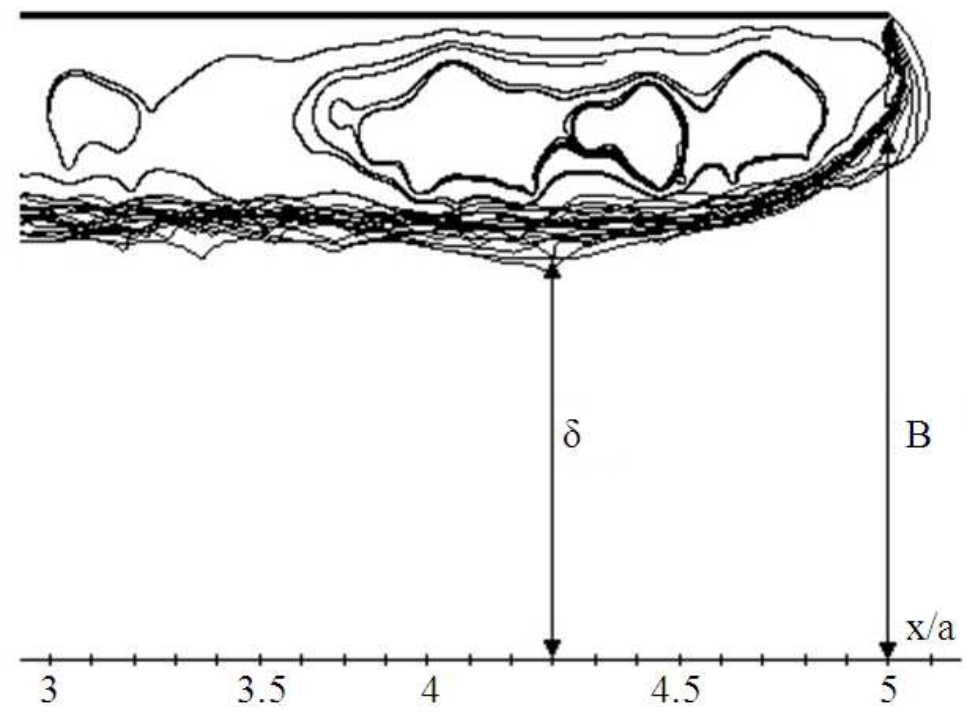

Fig. 3. The flow lines in the section of XOY (XOZ)

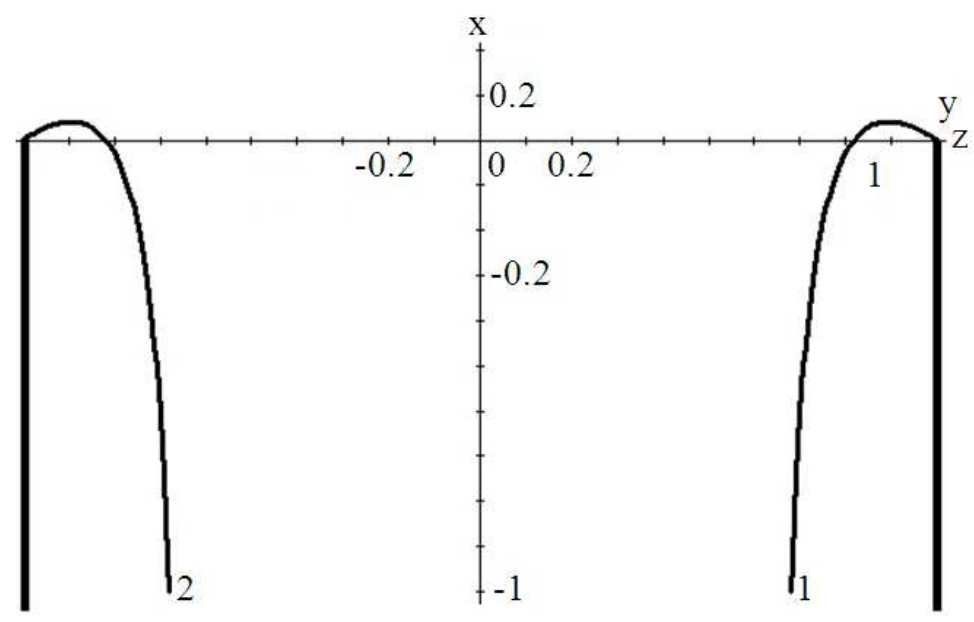

Fig. 4. Flow lines, built by the formula (8) 


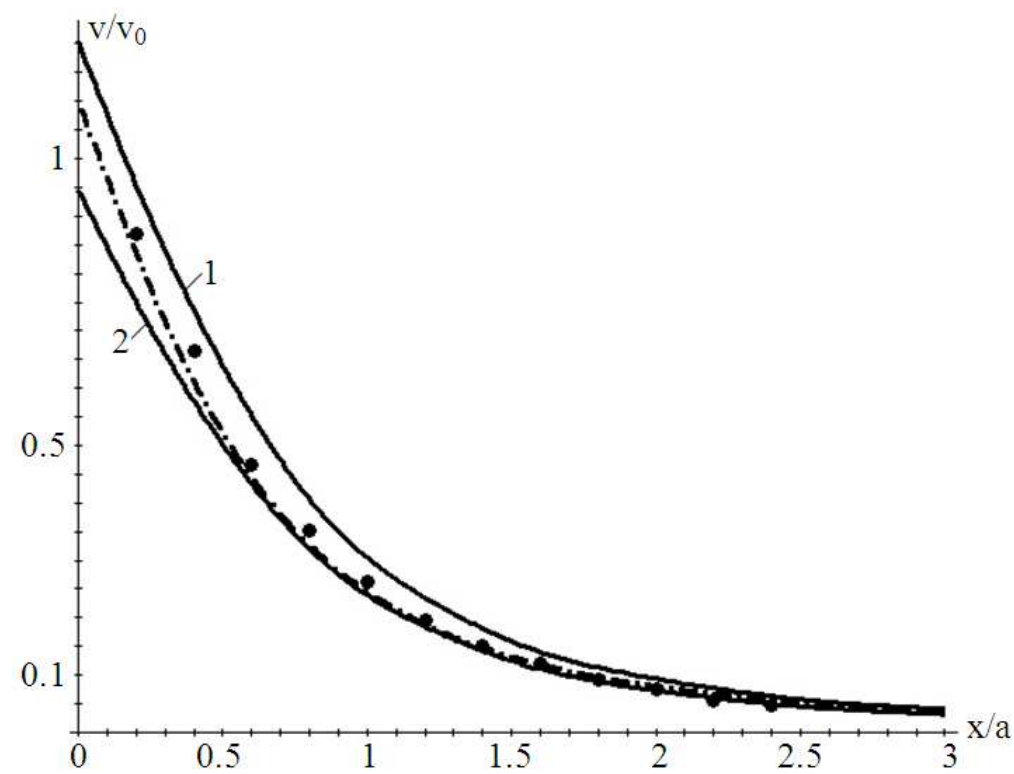

Fig. 5. Change of axial velocity with increasing distance from the entrance of the suction channel

The dash-dot line- this are calculations made by the formula of (Minko et al., 2010) for a round pipe $\mathrm{v} / \mathrm{v}_{0}=1.1\left(1-\frac{1}{\sqrt{1+0.655 /(\mathrm{x} / \mathrm{a})^{2}}}\right)$.

Circles-are results of experiment of (Wabeke, 2010; Logachev and Logachev, 2014) for a round pipe.

For a square tube a-is a half side of the square, or the same that radius of a circle inscribed in it. In this case the comparison occurs in the same locations. In case of equal speed $v_{0}$ in the suction, we have an excessive magnitude of the velocity (curve 1) for a square tube. However, this comparison is incorrect, since the flow in the given pipe is higher than in a circle one, by an amount, where a-side of the square. Therefore, speed value that was found for the square pipe should be referred to $(4 / \pi) v_{0}$. In this case, the volumetric flow rates of intake air will be the same for round and square tubes. Curve 2 in Fig. 2 shows, that the value of the axial velocity at the entrance of pipe into a square is lower than a circular one, i.e., it has a less range of square suction hole. Note that sometimes it is claimed about a big range of square suction pipe, but that comparison is performed at various points. Distance is measured in the hydraulic radius from the suction inlet; hole area, referred to the perimeter. For circular hole this value is $\mathrm{a} / 2$; for a square $\mathrm{a} / 4$. In a point lying close to hole rate of speed is naturally higher.
Let us note that in these formulas (9) it was assumed that a compressed section has a square shape.

This form have a little disturb on approaching to the corners of the cross section. Sectional Square of suction is $0.699 \alpha^{2}$, which is $4 \%$ more than if you consider it a square with an area equal to $0.672 \alpha^{2}$. The effective square of absorption in the submerged section at a distance of $0.8 \alpha$ from the entrance of the pipe is $0.50675 \alpha^{2}$. If we calculate local drag coefficient by the formula of Borda (Averkova et al., 2013c; Idelchik, 2008):

$$
\zeta=\left(1-\frac{\mathrm{a}^{2}}{\mathrm{~S}}\right)^{2}=\left(1-\frac{1}{0.507}\right)^{2}=0.95
$$

For practice this ratio is considered to be equal to 1 .

\section{CONCLUSION}

As a part of ideology of the method of discrete vortices in the non-stationary quasi-axisymmetric formulation, we constructed the mathematical model of separated flow at the inlet to the square aspirating pipe and its software-algorithmic implementation. We have determined the velocity field at the entrance to suction channel and a line of flow separation. The obtained results can be useful for designing of local exhaust ventilation of reduced energy consumption. 
Limitations of this study is that not considered viscosity of air at flow separation from of sharp edges of the suction duct.

Prospects for future research are to develop methods to increase the range of the suction hole that can be achieved, for example, by screening local ventilation supply air flow. This will give opportunity contamination capture with minimal energy consumption.

\section{ACKNOWLEDGEMENT}

The researchers are being supported by the Council for Grants of the President of the Russian Federation (projects MK-103.2014.1), RFBR (project number 1408-31069 mol_a) and Strategic Development Plan of BSTU named after. V. G. Shukhov.

\section{REFERENCES}

Averkova, O., A. Logachev, I. Logachev and K. Logachev, 2012. Modeling of gas separated flows at inlet of suction channels on the basis of stationary discrete vortices. Proceedings of the European Congress on Computational Methods in Applied Sciences and Engineering, (ACE' 12), e-Book Full Papers, pp: 20-20.

Averkova, O.A., V.Y. Zorya, I.N. Logachev and K.I. Logachev, 2010. Numerical simulation of air currents at the inlet to slot leaks of ventilation covers. Refractories Indust. Ceram., 51: 177-182. DOI: $10.1007 / \mathrm{s} 11148-010-9284-7$

Averkova, O.A., I.N. Logachev, K.I. Logachev and I.V. Khodakov, 2013a. About the modeling of detachable flows on the entrance of the round soaking-up branch pipe. Middle-East J. Scientific Res., 17: 1187-1193. DOI: 10.5829/idosi.mejsr.2013.17.08.7095

Averkova, O.A., A.E. Canar, K.I. Logachev and V.A. Uvarov, 2013b. Simulation of processes of ventilation in the confided area of trapezoidal form. Middle-East J. Scientific Res., 17: 1176-1180. DOI: 10.5829/idosi.mejsr.2013.17.08.7093

Averkova, O.A., I.N. Logachev, K.I. Logachev and A.K. Logachev, 2013c. The principles of separated flow at the inlet of the protruding duct with screens. TsAGI Sci. J., 44: 219-243.
Averkova, O.A., V.I. Belyaeva, K.I. Logachev, V.A. Uvarov and A.E. Canar, 2013d. About dynamics of clinker dust in an aspiration hideout. Middle-East J. Scientific Res., 17: 1181-1186. DOI: 10.5829/idosi.mejsr.2013.17.08.7094

Averkova, O.A., I.N. Logachev, K.I., Logachev and I.V. Khodakov. 2014. Modeling detached flows at the inlet to round suction flues with annular screens. Refractories Indust. Ceram., 54: 425-429. DOI: 10.1007/s11148-014-9625-z

Ginevsky, A.S. and A.I. Zhelannikov, 2009. Vortex wakes of Aircrafts. 1st Edn., Springer, Dordrecht, ISBN-10: 3642017606, pp: 169.

Idelchik, I.E., 2008. Handbook of Hydraulic Resistance. 4st Edn., Begell House, New York, ISBN: 978-156700-251-5, pp: 861.

Logachev, I.N. and K.I. Logachev, 2014. Industrial Air Quality and Ventilation: Controlling Dust Emissions. 1st Edn., CRC Press, Boca Raton, ISBN10: 9781482222166, pp: 417.

Logachev, I.N., K.I. Logachev and O.A. Averkova, 2010. Mathematical modeling of separated flows at inlet of a shielded flat duct. Computat. Meth. Programm., 11: 68-77.

Logachev, I.N., K.I. Logachev and O.A. Averkova, 2013. Methods and means of reducing the power requirements of ventilation systems in the transfer of free-flowing materials. Refract. Indus. Ceram., 54: 258-262. DOI: $10.1007 / \mathrm{s} 11148-013-9585-8$

Marshall, J.S and Li. Shuiqing, 2014. Adhesive Particle Flow: A Discrete-Element Approach. 1st Edn., Cambridge University Press, ISBN-13: 9781107032071 , pp: 394.

Minko, V.A. I.N. Logachev and K.I. Logachev, 2010. Dedusting ventilation, Vol. 2. Printing Office of the V.G. Shukhov State Technology University of Belgorod, Belgorod.

Schinzinger, R. and P.A.A. Laura, 2012. Conformal Mapping: Methods and Applications. 1st Edn., Dover Publications, New York, pp: 624.

Wabeke, R.L., 2010. Air Contaminants, Ventilation and Industrial Hygiene Economics: The Practitioner's Toolbox and Desktop Handbook. 1st Edn., CRC Press, Boca Raton, ISBN-10: 1466577908, pp: 445. 